\title{
Non-Markovian Dynamic of Gaussian Quantum Discord in Continuous-Variable Systems
}

\author{
X. LIU* AND W. WU \\ Department of Physics, School of Science, Wuhan University of Technology (WHUT), Wuhan, China \\ (Received January 29, 2014; in final form June 9, 2014) \\ We show the special dynamic characteristic of Gaussian quantum discord, exhibited by two-mode Gaussian \\ symmetric squeezed thermal states (STS) in continuous-variable (CV) systems with a common non-Markovian \\ environment, is definitely different from Markov processes. We demonstrate that Gaussian quantum discord can \\ be created whenever the information flow from environment back to the system. We also show that the rate of \\ decrease for Gaussian quantum discord is related to the coupling constant. We discover that the initial value of \\ Gaussian quantum discord is determined by the average number of thermal photons of the system.
}

DOI: 10.12693/APhysPolA.126.652

PACS: 03.65.Yz, 03.65.Ta, 03.65.Ud, 03.67.Mn

\section{Introduction}

Quantum correlations (QCs), seminally quantified by quantum discord (QD), are a fundamental resource for quantum information processing (QIP) [1]. In the last two decades, people believed that entanglement can characterize QCs, however with some new discovery people found that entanglement was only one kind of QCs and could not represent all nonclassical correlations [2]. Now a widely acceptable concept to measure QCs is quantum discord (QD) which was introduced by Ollivier and Zurek [3]. QD is defined as the mismatch between two natural quantum extensions of classically equivalent expression of the mutual information in a bipartite state.

With a variety of studies it has been known that QD has a significant application in deterministic quantum computation with one pure qubit (DQC1) $[4,5]$ and can be used to better understand the quantum phase transition $[6,7]$. It also can be used to measure the QCs between relatively accelerated observers $[8,9]$. In recently years the notion of discord is extended to the domain of bipartite quantum CV systems [10-11] in which QD is called GQD.

In the literature the dynamic of open quantum systems $[12,13]$ is often considered in a Markov process which is described by a master equation with Lindblad structure $[14,15]$. However, in realistic physical systems Lindblad master equations are derived just under a number of drastic simplifications. So many methods have been devoted to define non-Markovianity in open quantum systems $[16,17]$ and to quantify the degree of nonMarkovian behavior [18-20] recently.

In discrete systems some important results for QD in non-Markovian evolution have been mentioned in Refs. [21-23], but in CV systems few discuss has been made for GQD in non-Markovian environments. In this paper we analyze the dynamic of GQD in a CV system for a non-Markovian process. By evaluating the evolution

\footnotetext{
*e-mail: lxheroes@126.com
}

of GQD, we find that GQD can be created by the feature of non-Markovianity and we reveal the relationship between GQD and some important parameters.

The paper is structured as follows. In Sec. 2 the definition of GQD and its expression in STS are reviewed. In Sec. 3 the definition of non-Markovianity for a CV system that we use in this paper is described. In Sec. 4 we show the dynamic of GQD of a two-mode Gaussian symmetric STS in a common non-Markovian reservoir with zero temperature. Finally, In Sec. 5 we draw our conclusions.

\section{GQD of two-mode squeezed thermal states}

The quantum mutual information between two subsystems $A$ and $B$ in a state $\rho_{A B}$ is defined as

$$
I\left(\rho_{A B}\right)=S\left(\rho_{A}\right)+S\left(\rho_{B}\right)-S\left(\rho_{A B}\right),
$$

where $S(\rho)=-\operatorname{Tr}\left(\rho \log _{2} \rho\right)$ is the von Neumann entropy of an arbitrary state $\rho$ and $\rho_{A(B)}=\operatorname{Tr}_{B(A)} \rho_{A B}$ is the reduced density operator describing the state of $A(B)$. An other expression for the mutual information defined via a measurement-based conditional density operator is

$$
J_{A}\left(\rho_{A B}\right)=\max _{\left\{\prod_{i}\right\}}\left\{S\left(\rho_{A}\right)-\sum_{i} p_{i} S\left(\rho_{A \mid i}\right),\right.
$$

where $\left\{\prod_{i}\right\}$ denotes a complete set of positive operatorvalued measure (POVM) performed on the subsystem $B$ and $\rho_{A \mid i}=\operatorname{Tr}_{B}\left(\rho_{A B} \prod_{i}\right) / p_{i}$ is the remaining state of subsystem $A$ after the measurement with probability $p_{i}=\operatorname{Tr}_{A B}\left(\rho_{A B} \prod_{i}\right)$. The mismatch between (1) and (2) is defined as $A$ QD $[2,3]$

$$
D_{A}\left(\rho_{A B}\right)=I\left(\rho_{A B}\right)-J_{A}\left(\rho_{A B}\right) .
$$

Analogously, $B$ QD is defined where the roles of $A$ and $B$ are swapped.

Now a Gaussian version of QD is defined $[10,11]$ in an bipartite CV system. We restrict $\rho_{A B}$ to a two-mode Gaussian state whose covariance matrix can be transformed in a standard form

$$
\sigma_{A B}=\left(\begin{array}{cc}
A & C \\
C^{T} & B
\end{array}\right)
$$

with $A=\operatorname{diag}(a, a), B=\operatorname{diag}(b, b)$, and $C=\operatorname{diag}(c, d)$. The quantities $I_{1}=\operatorname{det} A, I_{2}=\operatorname{det} B, I_{3}=\operatorname{det} C$, and $I_{4}=\operatorname{det} \sigma$ are referred to as symplectic invariants 
for they are invariant under local symplectic transformations. Because of $J_{A}\left(\rho_{A B}\right)$ and $D_{A}\left(\rho_{A B}\right)$ are invariant under local unitary transformation $[2,3]$, we can extend $A \mathrm{QD}$ to the standard form of a general two-mode Gaussian state $[10,11]$ based on the minimization of the mismatch $I\left(\rho_{A B}\right)-J_{A}\left(\rho_{A B}\right)$ over single-mode generalized Gaussian measurements on subsystem $B$ as follow

$$
\begin{aligned}
& \left.C_{A}\left(\rho_{A B}\right)=h\left(\sqrt{I_{2}}\right)-h\left(\sqrt{d_{-}}\right)-h \sqrt{d_{+}}\right) \\
& \quad+\inf _{\sigma_{M}} h\left(\sqrt{\operatorname{det} \sigma_{A}}\right),
\end{aligned}
$$

where $h(x)=\left(x+\frac{1}{2}\right) \ln \left(x+\frac{1}{2}\right)-\left(x-\frac{1}{2}\right) \ln \left(x-\frac{1}{2}\right)$, $d_{ \pm}$are the symplectic eigenvalues of $\rho_{A B}$ which are expressed by $d_{ \pm}^{2}=\frac{\Delta \pm \sqrt{\Delta^{2}-4 I_{4}}}{2}$ with $\Delta=I_{1}+I_{2}+2 I_{3}$, and $\sigma_{M}$ is the covariance matrix of single-mode Gaussian states for Gaussian measurement on subsystem B, $\sigma_{A}=A-C\left(B+\sigma_{M}\right)^{-1} C^{T}[25]$ is the covariance matrix of the subsystem $A$ after measurement. In this paper, we will focus on the relevant subclass of two-mode STSs with generic state

$$
\rho=V(r) \rho_{1} \otimes \rho_{2} V^{+}(r),
$$

where $V(r)=e^{r\left(a^{+} b^{+}-a b\right)}$ is the two-mode squeezing operator and $\rho_{i}(i=1,2)$ is given by $\rho_{i}=\sum_{n} N_{i}^{n}(1+$ $\left.N_{i}\right)^{-n-1}|n\rangle_{i}\langle n|$ with $N_{i}$ is the average number of thermal photons. For the case of STS, we can obtain the diagonal matrix elements which define $A, B$, and $C$

$$
\begin{aligned}
& a=\left(N_{r}+\frac{1}{2}\right)+N_{1}\left(1+N_{r}\right)+N_{2} N_{r}, \\
& b=\left(N_{r}+\frac{1}{2}\right)+N_{2}\left(1+N_{r}\right)+N_{1} N_{r}, \\
& c=-d=\left(1+N_{1}+N_{2}\right) \sqrt{N_{r}\left(1+N_{r}\right)},
\end{aligned}
$$

where $N_{r}=\sinh ^{2} r$. The Gaussian $A$ QD for the generic bipartite STS then can be written as

$$
\begin{aligned}
& C_{A}\left(\rho_{A B}\right)=h\left(\sqrt{I_{2}}\right)-h\left(\sqrt{d_{-}}\right)-h\left(\sqrt{d_{+}}\right) \\
& \quad+h\left(\frac{\sqrt{I_{1}}+2 \sqrt{I_{1} I_{2}}+2 I_{3}}{1+2 \sqrt{I_{2}}}\right) .
\end{aligned}
$$

By exchanging $I_{1} \leftrightarrow I_{2}$, Gaussian $B$ QD is obtained where Gaussian measurements are performed on subsystem $A$. In the following part, we will use Gaussian $A$ QD as GQD.

\section{A non-Markovianity measure for continuous-variable systems}

Some measures have been introduced for detecting non-Markovianity of an evolution [16-20]. In this paper we use the measurement which is first introduced by Breuer et al. [8], and extended to $\mathrm{CV}$ systems by Vasile et al. [24]. In this measurement the information which flows between the system and its environment is treated as the key feature of non-Markovianity.

The fundamental idea of the measure is that a Markovian process tends to reduce the distinguishability between any two states continuously, contrarily a nonMarkovian process means the increasing of the distinguishability. The interpretation of this phenomenon is that when information flows from the system to its environment, the distinguishability of states is decrease and when it reverses, the distinguishability arises. So the measure is based on the trace distance [1] of two quantum states which describes the probability of successfully distinguishing the states. The trace distance is defined as

$$
D\left(\rho_{1}, \rho_{2}\right)=\frac{1}{2} \operatorname{tr}\left|\rho_{1}-\rho_{2}\right|
$$

where $|A|=\sqrt{A^{+} A}$. In a Markovian dynamic the trace distance is always monotonic decrease, namely

$$
D\left(\rho_{1}(\tau+t), \rho_{2}(\tau+t)\right) \leq D\left(\rho_{1}(t), \rho_{2}(t)\right) .
$$

When monotonicity is not satisfied it means that there are intervals of time for which the states become more distinguishable compared to previous instants, this characterizes a non-Markovian evolution. In a generic $\mathrm{CV}$ state there is no analytic expression for the trace distance, but replaceable signatures can be employed within the same spirit, such as the fidelity

$$
F\left(\rho_{1}, \rho_{2}\right)=\operatorname{tr} \sqrt{\sqrt{\rho_{1}} \rho_{2} \sqrt{\rho_{1}}} .
$$

Which is a good candidate of taking the role of the trace distance in a CV system. That is in a CV system,

$$
F\left(\rho_{1}(\tau+t), \rho_{2}(\tau+t)\right) \leq F\left(\rho_{1}(t), \rho_{2}(t)\right)
$$

holds for a Markovian process, whenever it fails there is a non-Markovian process.

In this paper we consider the dynamic described by the phenomenological equation with a single decay channel in Lindblad form with a time-dependent damping rate $\gamma(t)$ as follow

$$
\frac{\mathrm{d} \rho}{\mathrm{d} t}=\alpha \frac{\gamma(t)}{2}\left(2 a \rho a^{+}-a^{+} a \rho-\rho a^{+} a\right),
$$

where $a$ is the annihilation operator. The function $\gamma(t)$ determined by the spectral density $J(w)$ of the reservoir. Any Gaussian state evolving according to Eq. (15) remains Gaussian and the covariance matrix evolving in a zero-temperature environment (the average number of thermal photons for environment $N_{e}=0$ ) is as follow

$$
\sigma(t)=e^{-x(t)} \sigma(0)+\left[1-e^{-x(t)}\right] \frac{\mathrm{I}}{2},
$$

where $x(t)=\alpha \int_{0}^{t} 2 \gamma(s) \mathrm{d} s$, and $\alpha$ is a coupling constant. It has been proven $[18,24]$ that whenever $\gamma(t)<0$, Eq. (12) or (14) is not satisfied and we can say the evolution is non-Markovianity. So if there exists some negativity intervals of $\gamma(t)$, it means in the physical process Eq. (14) fails for certain times and then Eq. (15) describes a non-Markovian process.

\section{Behavior of Gauss discord with non-Markovianity}

In this section we study the behavior of GQD of a two-mode Gaussian STS in a common non-Markovian reservoir with zero-temperature which is described in the previous section. We analyze the case of symmetric STS in which $N_{1}=N_{2}$. In example, we consider the damping rate is 


$$
\gamma(t)=\frac{1}{2}\left\{\begin{array}{ll}
\mathrm{e}^{-t / 5} \sin t, & \text { if } t<5 \pi / 2 \\
\mathrm{e}^{-\pi / 2}, & \text { if } t \geq 5 \pi / 2
\end{array},\right.
$$

which is characterized by only one interval of negativity, $[\pi, 2 \pi]$ for non-Markovianity, $N_{1}=N_{2}=10$ and $r=2$. We plot the evolution of GQD with $\alpha=0.5$ in Fig. 1b. In contrast, the situation for a Markov process is exhibited in Fig. 1a with the damping rate is

$$
\gamma(t)=\frac{1}{2}\left\{\begin{array}{ll}
\mathrm{e}^{-t / 5} \sin t, & \text { if } t<\pi \\
\mathrm{e}^{-\pi / 2}, & \text { if } t \geq \pi
\end{array} .\right.
$$

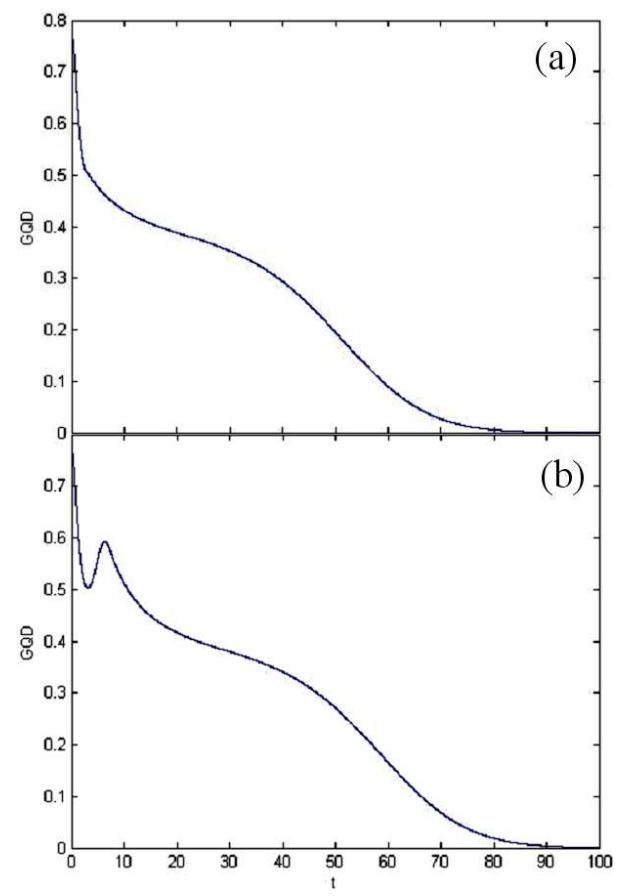

Fig. 1. Evolution of GQD in (a) Markov case and in (b) non-Markovian case with $N_{1}=N_{2}=10, r=2$ and $\alpha=0.5$

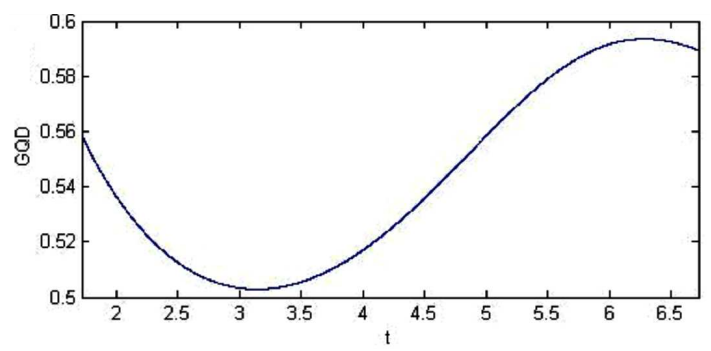

Fig. 2. The detail of the rising time of GQD in nonMarkovian case.

We can see that GQD monotonically decreases in a Markov process, but it is different in a non-Markov case. During the decay GQD has a rise suddenly and then decreases to zero eventually. By observing the rising time in details in Fig. 2, we find it is just the time when $\gamma(t)$ is negativity. We think that the physical explanation for the rise is the flow of information from the environment

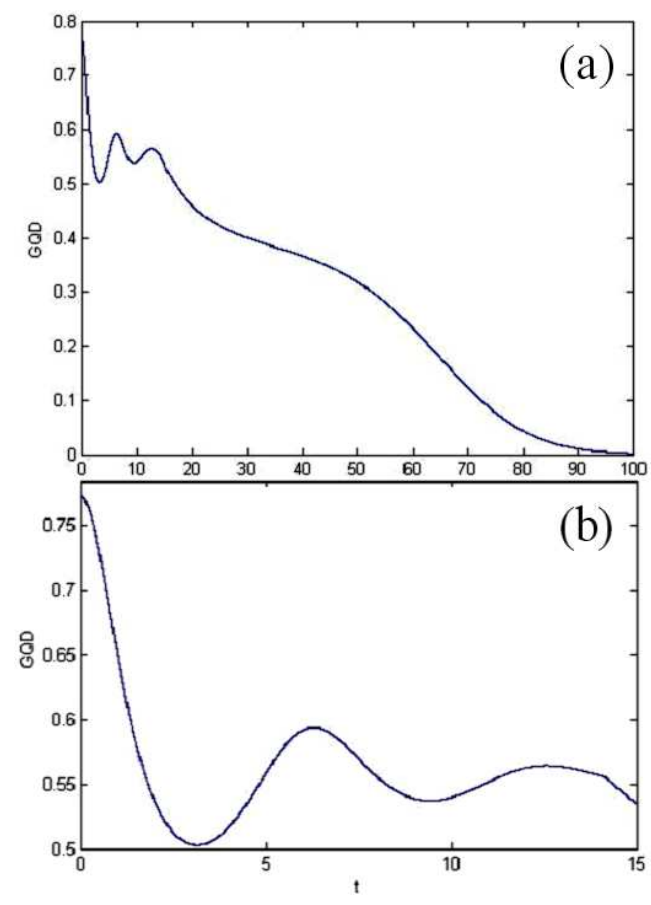

Fig. 3. Evolution of GQD with (a) $N_{1}=N_{2}=10$, $r=2, \alpha=0.5$ and (b) the detail of the rising time in non-Markovian case where $\gamma(t)$ is in the form of Eq. (19).

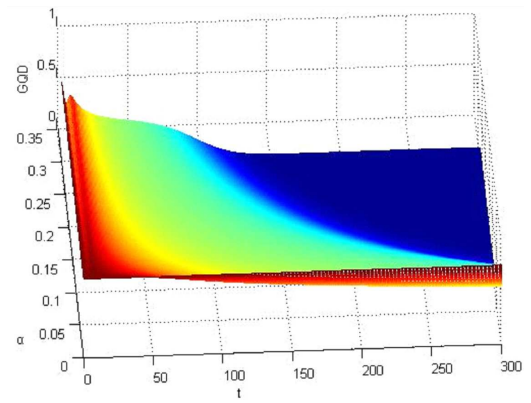

Fig. 4. Evolution of GQD as a function of $\alpha$ and $t$.

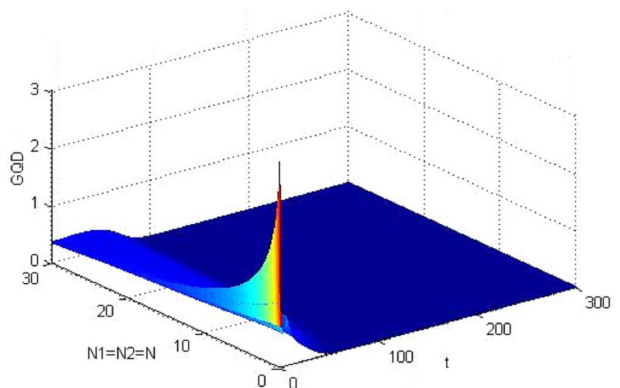

Fig. 5. Evolution of GQD as a function of $N_{i}$ and $t$. 
back to the system $[18,24]$. Then we adjust the damping rate

$$
\gamma(t)=\frac{1}{2} \times\left\{\begin{array}{ll}
\mathrm{e}^{-t / 5} \sin t, & \text { if } t<9 \pi / 2 \\
\mathrm{e}^{-\pi / 2}, & \text { if } t \geq 9 \pi / 2
\end{array},\right.
$$

which has two intervals of negativity, $[\pi, 2 \pi]$ and $[3 \pi, 4 \pi]$. In Fig. 3a we can see there are two rises for GQD during the course and the happening time which is showed in Fig. 3b is also the exactly time whenever $\gamma(t)$ is negativity. So we conclude that once the information flows back from environment to system which features nonMarkovianity of system, it causes a rise for GQD. In other word, quantum correlations are created due to nonMarkovianity.

We study the influence which is caused by the coupling constant $\alpha$ and the average number of thermal photons $N_{i}(i=1,2)$ in symmetric STS respectively. The evolution of GQD as a function of $\alpha$ and $t$ can be seen in Fig. 4 . We discover that GQD falls slower when $\alpha$ is smaller. In Fig. 5 where we consider $r=2, \alpha=0.5$ and $\gamma(t)$ is in the form of Eq. (17), we find that with the value of $N_{i}$ becomes larger, GQD gets a smaller initial value.

\section{Conclusions}

In this paper, the dynamic of GQD of a $\mathrm{CV}$ system in a non-Markovian process is studied. We have showed the behavior of GQD of a two-mode Gaussian symmetric STS in a common non-Markovian reservoir with zerotemperature. We have found that in a non-Markovian case GQD can be created during the evolution, which indicates that QCs can be produced in a non-Markovian environment. We have also found that the rising time is exactly whenever the information flows from the environment back to the system. The result will be very significant for a practical purpose. Finally we have revealed the influence of GQD which is caused by the coupling constant $\alpha$ and the average number of thermal photons $N_{i}$ respectively. We have found that $\alpha$ affects the rate of decrease and the initial value is associated with $N_{i}$.

\section{Acknowledgments}

This work is supported by "the Fundamental Research Funds for the Central Universities" under the project number 2013-Ia-032 and "the Fundamental Research Funds for the Central Universities (WUT:2014Ia-026)".

\section{References}

[1] M.A. Nielsen, I.L. Chuang, Quantum Computation and Quantum Information, Cambridge University Pub., Cambridge 2000.

[2] V. Vedral, Phys. Rev. Lett. 90, 050401 (2003).

[3] H. Ollivier, W.H. Zurk, Phys. Rev. Lett. 88, 017901 (2001).

[4] T. Yu, J.H. Eberly, Science 323, 598 (2009).

[5] A. Datta, A. Shaji, C.M. Caves, Phys. Rev. Lett. 100, 050502 (2008).

[6] R. Dillenschneider, Phys. Rev. B 78, 224413 (2008).

[7] M.S. Sarandy, Phys. Rev. A. 80, 022108 (2009).

[8] A. Shabani, D.A. Lidar, Phys. Rev. Lett. 102, 100402 (2009).

[9] A. Datta, Phys. Rev. A 80, 052304 (2009).

[10] P. Giorda, M. G. A. Paris, Phys. Rev. Lett. 105, 020503 (2010).

[11] G. Adesso, A. Datta, Phys. Rev. Lett. 105, 030501 (2010).

[12] H.P. Breuer, F. Petruccione, The Theory of Open Quantum Systems, Oxford University Pub., Oxford 2007.

[13] U. Weiss, Quantum Dissipative Systems, World Scientific, Singapore 2008.

[14] V. Gorini, A. Kossakowski, E. C. G. Sudarshan, J. Math. Phys. (N.Y.) 17, 821 (1976).

[15] G. Lindblad, Commun. Math. Phys. 48, 119 (1976).

[16] L. Mazzola, S. Maniscalco, J. Piilo, K.A. Suominen, B.M. Garraway, Phys. Rev. A 80, 012104 (2009).

[17] S. Maniscalco, S. Olivares, M.G.A. Paris, Phys. Rev. A 75, 062119 (2007).

[18] H.P. Breuer, E.M. Laine, J. Piilo, Phys. Rev. Lett. 103, 210401 (2009).

[19] M.M. Wolf, J. Eisert, T.S. Cubitt, J.I. Cirac, Phys. Rev. Lett. 101, 150402 (2008).

[20] A. Rivas, S.F. Huelga, M.B. Plenio, Phys. Rev. Lett. 105, 050403 (2010).

[21] B. Wang, Z.-Y. Xu, Z.-Q. Chen, M. Feng, Phys. Rev. A 81, 014101 (2010).

[22] F. F. Fanchini, T. Werlang, C.A. Brasil, L.G.E. Arruda, A.O. Caldeira, Phys. Rev. A 81, 052107 (2010).

[23] S. Alipour, A. Mani, A.T. Rezakhani, Phys. Rev. A 85, 052108 (2012).

[24] R. Vasile, S. Maniscalco, M.G.A. Paris, H.P. Breuer, J. Piilo, Phys. Rev. A 84, 052118 (2011).

[25] M. Takeoka, M. Sasaki, Phys. Rev. A 78, 022320 (2008). 